\title{
Simulation climatic model for time to flowering in wild chickpea
}

\author{
Andrey Ageev \\ SPbPU, St.Petersburg, Russia \\ andreyageev1@mail.ru \\ Abdullah Kahraman \\ Harran University, Sanliurfa, Turkey \\ kahraman55@hotmail.com \\ Sergey Nuzhdin \\ SPbPU, St.Petersburg, Russia \\ USC, LA, CA, USA \\ snuzhdin@usc.edu
}

\author{
Jens Berger \\ CSIRO, WA, Australia \\ Jens.Berger@csiro.au \\ Abdulkadir Aydogan \\ CRIFC, Ankara, Turkey \\ akadir602000@yahoo.com \\ Maria Samsonova \\ SPbPU, St.Petersburg, Russia \\ m.samsonova@spbstu.ru
}

\author{
Eric Bishop-von Wettberg \\ UVM, VT, USA \\ Eric.Bishop-Von-Wettberg@uvm.edu \\ Douglas Cook \\ UC Davis, CA, USA \\ drcook@ucdavis.edu \\ Konstantin Kozlov \\ SPbSPU, St.Petersburg, Russia \\ kozlov_kn@spbstu.ru \\ snuzhdin@usc.edu
}

\begin{abstract}
Accurate prediction of flowering time helps breeders to develop new varieties that can achieve maximal efficiency in changing climate. We have developed a methodology for construction a simulation model for time to flowering in which a function for daily progression of the plant from one to the next phenologic phase is obtained in analytic form by stochastic minimization. For demonstration, we build a simulation model for time to flowering in the dataset of wild chickpeas assembled recently.
\end{abstract}

Keywords - wild chickpea, simulation, climatic factors

Motivation and aim

\section{Motivation}

Chickpea (Cicer arietinum L.), which was originally domesticated in Southeastern Turkey, is the second most cultivated grain legume crop, grown in more than 50 countries of the world. Accurate prediction of crop flowering time is required for reaching maximal farm efficiency. Several models developed to accomplish this goal are based on deep knowledge of plant phenology, requiring large investment for every individual crop or new variety [1-2].

Aim

Mathematical modeling can be used to make better use of more shallow data and to extract information from it with higher efficiency. Earlier we have developed a non-linear regression model that predicted time to flowering based on climatic factors averaged over different periods of time [2]. Here to better interpret factors' impacts we build a simulation model for time to flowering that uses daily values of minimal and maximal temperature, solar radiation, precipitation and day length.

\section{Methods}

\section{Plant material}

Cultivars of chickpea, Cicer arietanum, are currently being improved by introgressing wild $C$. reticulatum biodiversity with very different flowering time requirements. The dataset consists of wild chickpea $(C$. reticulatum $\mathrm{L}$. and $C$. echinospermum). Accessions were collected at 21 sites in five regions in Turkey by von Wettberg et al. [3] and planted in climatically distinct sites in Turkey (Sanliurfa and Ankara). Being grown in contrasting environments the phenotype data on time to flowering is highly diverse - time to flowering ranges from
117 to 221 days. We build a model for samples sown in autumn (2174 samples).

\section{Climatic data}

The data on climatic conditions for each day in the period of field experiments were taken from publicly available site https://rp5.ru/Weather_in_the_world and NASA.

\section{Mathematical model}

The analytic representation of a function for daily progression of the plant from one to the next phenologic phase is build using a formal approach called Grammatical Evolution (GE) [4] and Differential Evolution Entirely Parallel (DEEP) method as stochastic minimization [5]. Consequently, a function is constructed as a superposition of arithmetic operations of expressions: $\mathrm{X}$, (X - Const), 1/(X - Const), where $X$ is a climatic factor and Const is a threshold value specific to each factor. DEEP is used to minimize the sum of squared differences between model solution and data.

\section{Results}

\section{Model adaptation}

The cross-validation and testing was performed to select the best and reliable model. The dataset was split into training, validation and test sets. The number of samples was 1482, 492 and 200, respectively. Optimization was performed with training set, then the model with a lowest score (18580.0) for the validation set was selected and run on a test set. According to Mann-Witney-Wilcoxon criterion the difference between mean errors for training and validation sets is not statistically significant $(\mathrm{P}=0.13)$. Consequently, the constructed model is validated. Mean deviation of the model solution from data for validation and test sets are 5.0 and 6.1 days, respectively. The analytic form of a function combines linear and multiplicative parts that is similar to the Soltani et al. model [1].

\section{Impacts of climatic factors}

To assess the impacts of climatic factors to the model we performed a permutation test with 100 permutations. The results are presented in Fig.1. The minimal temperature $T_{n}$ has the maximal impact to the model - over $85 \%$, the day length impact is $14.37 \%$. The impacts of maximal temperature, solar radiation and precipitation are almost negligible $-0.02 \%, 0.08 \%$ and $0 \%$, respectively. Day length has high impact on long day plants such as wild chickpea. 
The high impact of minimal temperature on time to flowering emphasizes the importance of vernalization.

The obtained results show that developed methodology can be used to model wild chickpea growth.

\section{ACKNOWLEDGMENT}

Supported by the RSCF (16-16-00007).

\section{REFERENCES}

[1] Soltani, A., and Sinclair, T.R. (2011). A simple model for chickpea development, growth and yield. Field Crops Research 124, 252-260.

[2] Kozlov, K., Singh, A., Berger, J., Wettberg, E.B., Kahraman, A., Aydogan, A., Cook, D., Nuzhdin, S., and Samsonova, M. (2019)
Nonlinear regression models for time to flowering in wild chickpea combine genetic and climatic factors. BMC Plant Biology 19, 94.

[3] von Wettberg, E.J.B., Chang, P.L., Başdemir, F., CarrasquilaGarcia, N., Korbu, L.B., Moenga, S.M., Bedada, G., Greenlon, A., Moriuchi, K.S., Singh, V., et al. (2018) Ecology and genomics of an important crop wild relative as a prelude to agricultural innovation. Nature Communications 9.

[4] O'Neill, M., and Ryan, C. (2001). Grammatical evolution. IEEE Transactions on Evolutionary Computation 5, 349-358.

[5] Kozlov, K., Samsonov, A.M., and Samsonova, M. (2016). A software for parameter optimization with Differential Evolution Entirely Parallel method. PeerJ Computer Science 2, e74. 